Изв. АН Эстонии. Физ. Матем., 1989, 38, № 4, 379-389

УдК 535.55

В. ШАРАФУТДИНОВ

\title{
О МЕТОДЕ ИНТЕГРАЛЬНОЙ ФОТОУПРУГОСТИ В СЛУЧАЕ СЛАБОЙ ОПТИЧЕСКОЙ АНИЗОТРОПИИ
}

\author{
(Представил Х. Абен)
}

Из всех известных поляризационно-оптических методов исследования напряжений метод интегральной фотоупругости $\left[^{1}\right]$ выгодно выделяется простотой и точностью измерений, но приводит к сложным математическим постановкам, большинство из которых еще ждут своего решения. В настоящей работе рассматривается одна из таких задач.

Если в пространстве имеется напряженная среда, характеризуемая тензором напряжений $\sigma$, то в случае слабой оптической анизотропии метод интегральной фотоупругости, как показано в $\left[{ }^{2}\right],\left[{ }^{3}\right]$, позволяет вдоль любой прямой $\pi$ измерить два числа

$L(\sigma, \pi)=\int \sigma_{Y Z}(X, 0,0) d X ; \quad S(\sigma, \pi)=\int\left(\sigma_{Y Y}-\sigma_{Z Z}\right)(X, 0,0) d X$,

где $X Y Z$ - прямоугольная система координат, относительно которой $\pi$ задается уравнениями $Y=Z=0$.

Технические условия проведения измерений накладывают определенные ограничения на семейство прямых $\pi$, вдоль которых можно измерить интегралы (1). Наиболее удобной с точки зрения технической реализации является ситуация, когда измерение интегралов (1) производится вдоль горизонтальных прямых, т. е. тех прямых, которые в некоторой (лабораторной) системе координат $x y z$ параллельны плоскости $z=0$. В связи с этим в настоящей работе рассматривается математическая постановка вопроса о том, насколько однозначно тензор напряжений определяется интегралами (1), измеренными вдоль всех горизонтальных прямых, лежащих в слое $a<z<b$. При этом предполагаем, что тензор напряжений удовлетворяет лишь уравнениям равновесия, и не прибегаем к рассмотрению деформаций. Поэтому выводы настоящей работы справедливы для любой модели (упругой, термоупругой, вязкоупругой и пр.) сплошной напряженной среды. Важно лишь предположение о том, что тензор диэлектрической проницаемости линейно зависит от тензора напряжений и при этом справедливо приближение слабой оптической анизотропии.

Будем считать, что напряженная среда заполняет цилиндрическую область $G=D \times(a, b)=\{(x, y, z) \mid(x, y) \in D, a<z<b\}$, где $D$ - двумерная область на плоскости переменных $(x, y)$, ограниченная строго выпуклой, замкнутой $C^{1}$-гладкой кривой $\gamma$. Пусть $H=\gamma \times(a, b)=$ $=\{(x, y, z) \mid(x, y) \in \gamma, a<z<b\}$ - боковая поверхность циличдра $G$. Будем считать, что компоненты тензора напряжений $\sigma$ имеют непрерывные вторые производные в $G$, а их первые производные непрерывны вплоть до боковой поверхности, т. е.

$$
\sigma \in C^{2}(G) \cap C^{1}(G \cup H) .
$$

Предполагаем, что в области $G$ справедливы уравнения равновесия (считаем, что объемные силы отсутствуют): 


$$
\begin{aligned}
& \frac{\partial \sigma_{x x}}{\partial x}+\frac{\partial \sigma_{x y}}{\partial y}+\frac{\partial \sigma_{x z}}{\partial z}=0, \\
& \frac{\partial \sigma_{y x}}{\partial x}+\frac{\partial \sigma_{y y}}{\partial y}+\frac{\partial \sigma_{y z}}{\partial z}=0 \\
& \frac{\partial \sigma_{z x}}{\partial x}+\frac{\partial \sigma_{z y}}{\partial y}+\frac{\partial \sigma_{z z}}{\partial z}=0,
\end{aligned}
$$

a на боковой поверхности цилиндра $G$ отсутствуют внешние нагрузки, т. е. при $(x, y, z) \in H$

$$
\begin{aligned}
& \sigma_{x x} n_{x}+\sigma_{x y} n_{y}=0, \\
& \sigma_{y x} n_{x}+\sigma_{y y} n_{y}=0, \\
& \sigma_{z x} n_{x}+\sigma_{z y} n_{y}=0,
\end{aligned}
$$

где $n=\left(n_{x}, n_{y}\right)$ - единичный вектор внешней нормали к $\gamma$. Для справедливости некоторых из наших выводов краевые условия (4) не существенны; это будем оговаривать в дальнейшем. Для удобства будем считать тензор $\sigma$ определенным во всей полосе $\{(x, y, z) \mid a<z<b\}$, положив $\sigma=0$ вне $G \cup H$.

Семейство горизонтальных прямых в пространстве является трехпараметрическим, но нам будет удобнее использовать 4 параметра. Обозначим через $\pi\left(x_{0}, y_{0}, \alpha, z_{0}\right)$ прямую, задаваемую параметрическими уравнениями

$$
x=x_{0}+t \cos \alpha, \quad y=y_{0}+t \sin \alpha, \quad z=z_{0} .
$$

Отметим, что $\pi(x, y, \alpha, z)$ фактически зависит от трех параметров $(x \sin \alpha-y \cos \alpha, \alpha, z)$. В результате измерения интегралов (1) вдоль всех горизонтальных прямых получаем две функции, определенные при $a<z<b$

$$
L_{\sigma}(x, y, \alpha, z)=L(\sigma, \pi(x, y, \alpha, z)) ; \quad S_{\sigma}(x, y, \alpha, z)=S(\sigma, \pi(x, y, \alpha, z)) .
$$

В настоящей работе получены следующие три основных результата.

1) Функции $L_{\sigma}$ и $S_{\sigma}$ зависимы, а именно $S_{\sigma}(x, y, \alpha, z)$ выражается через $L_{\sigma}(x, y, \alpha, z)$ и $S_{\sigma}\left(x, y, \alpha, z_{0}\right)$.

$2)$ Компонента $\sigma_{z z}(x, y, z)$ однозначно определяется функциями $L_{\sigma}(x, y, \alpha, z)$ и $S_{\sigma}(x, y, \alpha, z)$. Указаны явные процедуры определения $\sigma_{z z}$ по $L_{\sigma}$ и $S_{\sigma}$.

3) Никакой другой, кроме $\sigma_{z z}$, информации о поле напряжений $\sigma$ по функциям $L_{\sigma}$ и $S_{\sigma}$ определить нельзя. Точнее говоря, справедливо следующее утверждение. Если два тензорных поля $\sigma^{1}$ и $\sigma^{2}$ удовлетворяют (2), (3), (4) и если $\sigma_{z z}^{1} \equiv \sigma_{z z}^{2}$, то $L_{\sigma^{1}} \equiv L_{\sigma^{2}}, S_{\sigma^{1}} \equiv S_{\sigma^{2}}$.

В системе координат $X Y Z$, связанной с $x y z$ формулами перехода

$$
x=X \cos \alpha-Y \sin \alpha+x_{0}, \quad y=X \sin \alpha+Y \cos \alpha+y_{0}, \quad z=Z+z_{0},
$$

прямая $\pi\left(x_{0}, y_{0}, \alpha, z_{0}\right)$ задается уравнениями $Y=Z=0$, а компоненты тензора $\sigma$ относительно этих систем связаны соотношениями

$\sigma_{Y Y}(t, 0,0)=\sigma_{x x}\left(\bar{x}_{0}+\bar{\xi} t\right) \sin ^{2} \alpha-2 \sigma_{x y}\left(\bar{x}_{0}+\bar{\xi} t\right) \cos \alpha \sin \alpha+\sigma_{y y}\left(\bar{x}_{0}+\bar{\xi} t\right) \cos ^{2} \alpha$,

$$
\begin{gathered}
\sigma_{Y Z}(t, 0,0)=-\sigma_{x z}\left(\bar{x}_{0}+\bar{\xi} t\right) \sin \alpha+\sigma_{y z}\left(\bar{x}_{0}+\bar{\xi} t\right) \cos \alpha, \\
\sigma_{Z Z}(t, 0,0)=\sigma_{z z}\left(\bar{x}_{0}+\bar{\xi} t\right),
\end{gathered}
$$

в которых мы положили для краткости $\bar{x}_{0}=\left(x_{0}, y_{0}, z_{0}\right), \bar{\xi}=$ $=(\cos \alpha, \sin \alpha, 0)$. Подставив эти выражения в $(1)$, получим 
$L_{\sigma}(x, y, \alpha, z)=\int_{-\infty}^{\infty}\left[-\sigma_{x z}(\bar{x}+\bar{\xi} t) \sin \alpha+\sigma_{y z}(\bar{x}+\bar{\xi} t) \cos \alpha\right] d t$,

$$
S_{\sigma}(x, y, \alpha, z)=\int_{-\infty}^{\infty}\left[\left(\sigma_{x x}-\sigma_{z z}\right)(\bar{x}+\xi t) \sin ^{2} \alpha-\right.
$$

$$
\left.-2 \sigma_{x y}(\bar{x}+\bar{\xi} t) \cos \alpha \sin \alpha+\left(\sigma_{y y}-\sigma_{z z}\right)(\bar{x}+\bar{\xi} t) \cos ^{2} \alpha\right] d t .
$$

Зафиксируем $z_{0}\left(a<z_{0}<b\right)$ и определим на плоскости $z=z_{0}$ векторное поле $u=\left(u_{x}, u_{y}\right)$, положив

$$
u_{x}\left(x, y, z_{0}\right)=\sigma_{y z}\left(x, y, z_{0}\right) ; \quad u_{y}\left(x, y, z_{0}\right)=-\sigma_{x z}\left(x, y, z_{0}\right) .
$$

Тогда соотношение (5) можно переписать в виде

$$
L_{\sigma}\left(x, y, \alpha, z_{0}\right)=I u\left(x, y, \alpha, z_{0}\right),
$$

где

$$
\begin{gathered}
I u\left(x, y, \alpha, z_{0}\right)=\int_{-\infty}^{\infty}\left[u_{x}\left(x+t \cos \alpha, y+t \sin \alpha, z_{0}\right) \cos \alpha+\right. \\
\left.+u_{y}\left(x+t \cos \alpha, y+t \sin \alpha, z_{0}\right) \sin \alpha\right] d t
\end{gathered}
$$

Аналогично, если на плоскости $z=z_{0}$ определить симметричное тензорное поле $v=\left(v_{x x}, v_{x y}, v_{y y}\right)$, положив

$$
\begin{aligned}
& v_{x x}\left(x, y, z_{0}\right)=\left(\sigma_{y y}-\sigma_{z z}\right)\left(x, y, z_{0}\right), \\
& v_{x y}\left(x, y, z_{0}\right)=-\sigma_{x y}\left(x, y, z_{0}\right), \\
& v_{y y}\left(x, y, z_{0}\right)=\left(\sigma_{x x}-\sigma_{z z}\right)\left(x, y, z_{0}\right),
\end{aligned}
$$

то соотношение (6) можно переписать в виде

$$
S_{\sigma}\left(x, y, \alpha, z_{0}\right)=I v\left(x, y, \alpha, z_{0}\right) \text {, }
$$

где

$$
\begin{gathered}
I v\left(x, y, \alpha, z_{0}\right)=\int_{-\infty}^{\infty}\left[v_{x x}(\bar{x}) \cos ^{2} \alpha+2 v_{x y}(\bar{x}) \cos \alpha \sin \alpha+\right. \\
\left.+v_{y y}(\bar{x}) \sin ^{2} \alpha\right]_{\bar{x}=\left(x+t \cos \alpha, y+t \sin \alpha, z_{0}\right)} d t .
\end{gathered}
$$

Оператор $I$, определенный на тензорных полях степени 1 и 2 формулами (9) и (12) соответственно, называется лучевым преобразованием. Оно подробно изучалось в $\left[{ }^{4}\right],\left[{ }^{5}\right],\left[{ }^{6}\right]$. В частности в $\left[{ }^{4}\right],\left[{ }^{5}\right]$ доказано, что для тензорного поля $u$ интегральная информация $I u$ и локальная информация $\mathbb{W} u$ однозначно определяют друг друга, где $W$ - некоторый дифференциальный оператор, получивший в $\left[{ }^{4}\right]$ название оператора Сен-Венана. В $\left[{ }^{6}\right],\left[{ }^{7}\right]$ получена явная формула, выражающая $W u$ через $\mathrm{Iu}$. В помещенном ниже приложении эта формула приведена в виде, приспособленном для целей настоящей работы.

Для тензорного поля $u=\left(u_{x}, u_{y}\right)$ степени $1 \quad W_{D} u=\frac{\partial u_{y}}{\partial x}-\frac{\partial u_{x}}{\partial y}$ (по поводу обозначения $W_{D}$ см. приложение). Подставляя сюда выражение (7) для $u_{x}, u_{y}$, приходим к выводу, что функция

$$
l_{\sigma}(x, y, z)=-\frac{\partial \sigma_{x z}}{\partial x}-\frac{\partial \sigma_{y z}}{\partial y}
$$

однозначно определяется по функции $L_{\sigma}(x, y, \alpha, z)$. Явное выражение $l_{\sigma}$ через $L_{\sigma}$ вытекает из приводимых ниже формул $(40)-(42)$ с учетом равенств (8) и $l_{\sigma}=W_{D} u$. Аналогично, для тензорного поля $v=$ $=\left(v_{x x}, v_{x y}, v_{y y}\right)$ степени 2 


$$
W_{D} v=2 \frac{\partial^{2} v_{x y}}{\partial x \partial y}-\frac{\partial^{2} v_{x x}}{\partial y^{2}}-\frac{\partial^{2} v_{y y}}{\partial x^{2}}
$$

Подставляя сюда выражения (10) для $v_{i j}$, получаем, что функция

$$
s_{\sigma}(x, y, z)=\frac{\partial^{2}\left(\sigma_{z z}-\sigma_{x x}\right)}{\partial x^{2}}+\frac{\partial^{2}\left(\sigma_{z z}-\sigma_{y y}\right)}{\partial y^{2}}-2 \frac{\partial^{2} \sigma_{x y}}{\partial x \partial y}
$$

однозначно определяется функцией $S_{\sigma}(x, y, \alpha, z)$. Явное выражение $s_{\sigma}$ через $S_{\sigma}$ вытекает из приводимых ниже формул $(47)-(49)$ с учетом равенств (11) и $S_{\sigma}=W_{D} v$.

Вспомним, что б удовлетворяет уравнениям равновесия (3). В силу $\left(3_{3}\right)$ уравнение (13) эквивалентно следующему

$$
\frac{\partial \sigma_{z z}}{\partial z}=l_{\sigma}
$$

Если уравнение $\left(3_{1}\right)$ продифференцировать по $x$, $\left(3_{2}\right)-$ по $y$ и полученные соотношения прибавить к (14), то придем к уравнению

$$
\frac{\partial^{2} \sigma_{z z}}{\partial x^{2}}+\frac{\partial^{2} \sigma_{z z}}{\partial y^{2}}+\frac{\partial}{\partial z}\left(\frac{\partial \sigma_{x z}}{\partial x}+\frac{\partial \sigma_{y z}}{\partial y}\right)=S_{\sigma}
$$

которое в силу (13) эквивалентно уравнению

$$
\left(\frac{\partial^{2}}{\partial x^{2}}+\frac{\partial^{2}}{\partial y^{2}}\right) \sigma_{z z}=\frac{\partial l_{\sigma}}{\partial z}+s_{\sigma}
$$

Сравнивая (15) и (16), мы видим, что $l_{\sigma}$ и $s_{\sigma}$ связаны между собой соотношением

$$
\left(\frac{\partial^{2}}{\partial x^{2}}+\frac{\partial^{2}}{\partial y^{2}}-\frac{\partial^{2}}{\partial z^{2}}\right) l_{\sigma}=\frac{\partial s_{\sigma}}{\partial z} .
$$

Отметим, что уравнения (15)-(17) получены нами без использования краевых условий (4). Теперь, используя эти условия, установим, что значение $\sigma_{z z}$ на боковой поверхности $H$ цилиндра $G$ определяется по функции $S_{\sigma}$. Для этого возьмем две близкие точки $\bar{x}_{0}=\left(x_{0}, y_{0}, z_{0}\right)$ и $\bar{x}_{0}{ }^{\prime}=\left(x_{0}{ }^{\prime}, y_{0}{ }^{\prime}, z_{0}\right)$, принадлежащие $H$, и обозначим через $\pi\left(\bar{x}_{0}, \bar{x}_{0}{ }^{\prime}\right)$ горизонтальную прямую, проходящую через $\bar{x}_{0}$ и $\bar{x}_{0}{ }^{\prime}$. Согласно (6)

$$
\begin{aligned}
& S\left(\sigma, \pi\left(\bar{x}_{0}, \bar{x}_{0}^{\prime}\right)\right)=\int_{0}^{\left|\bar{x}_{0}-\bar{x}_{0}^{\prime}\right|}\left[\left(\sigma_{x x}-\sigma_{z z}\right)\left(\bar{x}_{0}+\bar{\xi} t\right) \xi_{y}^{2}-\right. \\
& \left.-2 \sigma_{x y}\left(\bar{x}_{0}+\bar{\xi} t\right) \xi_{x} \xi_{y}+\left(\sigma_{y y}-\sigma_{z z}\right)\left(\bar{x}_{0}+\bar{\xi} t\right) \xi_{x}^{2}\right] d t,
\end{aligned}
$$

где $\bar{\xi}=\left(\xi_{x}, \xi_{y}, 0\right)=\frac{\bar{x}_{0}^{\prime}-\bar{x}_{0}}{\left|\bar{x}_{0}^{\prime}-\bar{x}_{0}\right|}$. Устремим точку $\bar{x}_{0}^{\prime}$ к $\bar{x}_{0}$, тогда вектор $\bar{\xi}$ стремится к $\left(\tau_{x}, \tau_{y}, 0\right)$, где $\tau=\left(\tau_{x}, \tau_{y}\right)$ - единичный касательный вектор к кривой $\gamma$ в точке $\left(x_{0}, y_{0}\right)$. Применяя к интегралу (18) теорему о среднем значении, получнм

$$
\begin{gathered}
\lim _{\bar{x}_{\sigma}^{\prime} \leftrightarrow \bar{x}_{0}} \frac{S\left(\sigma, \pi\left(\bar{x}_{0}, \bar{x}_{0}^{\prime}\right)\right)}{\left|\bar{x}_{0}^{\prime}-\bar{x}_{0}\right|}=\sigma_{x x}\left(\bar{x}_{0}\right) \tau_{y}^{2}-2 \sigma_{x y}\left(\bar{x}_{0}\right) \tau_{x} \tau_{y}+ \\
+\sigma_{y y}\left(\bar{x}_{0}\right) \tau_{x}^{2}-\sigma_{z z}\left(\bar{x}_{0}\right) .
\end{gathered}
$$

Касательный вектор $\tau=\left(\tau_{x}, \tau_{y}\right)$ к $\gamma$ выражается через нормальный вектор $n=\left(n_{x}, n_{y}\right)$ по формулам $\tau_{x}=-n_{y}, \tau_{y}=n_{x}$. Подставив эти выражения в (19) и воспользовавшись $\left(4_{1}\right),\left(4_{2}\right)$, убедимся в справедливости равенства 


$$
\sigma_{z z}(\bar{x})=-\lim _{\bar{x}^{\prime} \rightarrow x} \frac{S\left(\sigma, \pi\left(\bar{x}, \bar{x}^{\prime}\right)\right)}{\left|\bar{x}^{\prime}-\bar{x}\right|} \quad\left(\bar{x}, \bar{x}^{\prime} \in H ; z=z^{\prime}\right) .
$$

Отметим, что это соотношение обобщает один из результатов работы $\left[{ }^{8}\right]$, в которой оно получено в осесимметричном случае.

Соотношения (15), (16), (20) позволяют утверждать, что функции $L_{\sigma}, S_{\sigma}$ однозначно определяют компоненту $\sigma_{z z}$. Что касается численных методов определения $\sigma_{z z}$, то в силу переопределенности системы (15), (16) здесь возможны рзличные варианты. Опишем вкратце два из них.

1) Фиксируем $z_{0}\left(a<z_{0}<b\right)$. Пользуясь измеренными значениями $S_{\sigma}\left(x, y, \alpha, z_{0}\right)$, находим на основании (20) значения $\sigma_{z z}(\bar{x})$ при $\bar{x}=$ $=\left(x, y, z_{0}\right) \in H$. Применяя к функциям $L_{\sigma}(x, y, \alpha, z)$ и $S_{\sigma}\left(x, y, \alpha, z_{0}\right)$ процедуры обращения лучевого преобразования, описанные в приводимом ниже приложении, находим функции $l_{\sigma}(x, y, z)$ и $s_{\sigma}\left(x, y, z_{0}\right)$. Pacсматривая (16) при фиксированном $z_{0}$ как уравнение Пуассона в области $D$ относительно функции $\sigma_{z z}\left(x, y, z_{0}\right)$ и используя найденные граничные значения $\left.\sigma_{z z}\left(x, y, z_{0}\right)\right|_{(x, y) \notin v}$, решаем задачу Дирихле и находим $\sigma_{z z}\left(x, y, z_{0}\right)$. Интегрируя $(15)$, определяем, исходя из $l_{\sigma}(x, y, z)$ и $\sigma_{z z}\left(x, y, z_{0}\right)$, функцию $\sigma_{z z}(x, y, z)$.

2) Пользуясь измеренными значениями $S_{\sigma}(x, y, \alpha, z)$, находим на основании (20) $\left.\sigma_{z z}\right|_{H}$. Применяя к $L_{\sigma}(x, y, \alpha, z)$ и $S_{\sigma}(x, y, \alpha, z)$ процедуры обращения лучевого преобразования, находим функции $l_{\sigma}(x, y, z)$ и $s_{\sigma}(x, y, z)$. Решая при каждом фиксированном $z$ задачу Дирихле для уравнения Пуассона $(16)$, находим $\sigma_{z z}(x, y, z)$.

Первый описанный метод требует, по сравнению со вторым, гораздо меньшего объема вычислений, поскольку в нем задачу Дирихле и задачу обращения лучевого преобразования для тензорного поля степени 2 нужно решать лишь один раз (при $z=z_{0}$ ). Таким образом, в первом методе основной объем вычислений приходится на процедуру обращения лучевого преобразования векторного поля (вычисления $l_{\text {व }}$ через $L_{\sigma}$ ), которую надо повторять многократно (для каждого значения $z$ ). Единственным, по мнению автора, недостатком первого метода является то, что ошибка определения $\sigma_{z z}(x, y, z)$ может накапливаться по мере удаления от сечения $z=z_{0}$, в то время как во втором методе все горизонтальные сечения равноправны. При вычислении по второму методу можно использовать соотношение (17) для контроля правильности определения $l_{\sigma}$ и $s_{\sigma}$. Возможны различные комбинации этих двух методов.

Докажем, что никакой другой, кроме $\sigma_{z z}$, информации о поле $\sigma$ по функциям $L_{\sigma}$ и $S_{\sigma}$ определить нельзя. Пусть тензорные поля $\sigma^{1} ; \sigma^{2}$ удовлетворяют $(2),(3),(4)$ и $\sigma_{z z}^{1} \equiv \sigma_{z z}^{2}$. Тогда их разность $\sigma=\sigma^{1}-\sigma^{2}$ удовлетворяет (2), (3), (4) и

Нужно показать, что

$$
\sigma_{z z} \equiv 0
$$

$$
\begin{aligned}
& L_{\sigma} \equiv 0, \\
& S_{\sigma} \equiv 0 .
\end{aligned}
$$

Зафиксируем $z_{0}$ и определим на плоскости $z=z_{0}$ тензорные поля $u, v$ степени 1 и 2 соответственно формулами (7) и (10). Тогда будут справедливы равенства (8) и (11). Поэтому для доказательства (22), (23) достаточно установить, что $I u \equiv 0, I v \equiv 0$. В силу результатов, полученных в $\left[{ }^{4}\right],\left[{ }^{5}\right]$, для этого, в свою очередь, достаточно установить, что

$$
\begin{aligned}
& W u=0, \\
& W v=0,
\end{aligned}
$$

где W- оператор Сен-Венана. 
Согласно приведенной ниже формуле (39)

$$
W u=\frac{\partial u_{y}}{\partial x}-\frac{\partial u_{x}}{\partial y}+\left(u_{x} n_{y}-u_{y} n_{x}\right) \delta_{\gamma}
$$

где $\delta_{\gamma}-\delta$-функция, сосредоточенная на кривой $\gamma, n=\left(n_{x}, n_{y}\right)-$ единичный вектор внешней нормали к $\gamma$. Подставляя в (26) выражения (7) для $u_{x}, u_{y}$, получим

$$
W u=-\left(\frac{\partial \sigma_{x z}}{\partial x}+\frac{\partial \sigma_{y z}}{\partial y}\right)+\left(\sigma_{x z} n_{x}+\sigma_{y z} n_{y}\right) \delta_{\gamma}
$$

Используя $\left(3_{3}\right)$, это можно переписать в виде

$$
W u=\frac{\partial \sigma_{z z}}{\partial z}+\left(\sigma_{x z} n_{x}+\sigma_{y z} n_{y}\right) \delta_{\gamma}
$$

Сравнивая это равенство с $\left(4_{3}\right)$ и (21), приходим к (24). Согласно приведенным ниже формулам (44)-(46)

$$
W v=W_{D} v+W_{\gamma} v
$$

где

$$
W_{D} v=2 \frac{\partial^{2} v_{x y}}{\partial x \partial y}-\frac{\partial^{2} v_{x x}}{\partial y^{2}}-\frac{\partial^{2} v_{y y}}{\partial x^{2}}
$$

a $W_{\gamma} v$ - обобщенная функция с носителем на кривой $\gamma$, которая определяется равенством $(\varphi(x, y)$ - произвольная гладкая функция на плоскости)

$$
\begin{gathered}
\left\langle W_{\gamma} v, \varphi\right\rangle=\oint_{\nu}\left(\frac{\partial v_{x x}}{\partial y} n_{y}+\frac{\partial v_{y y}}{\partial x} n_{x}-\frac{\partial v_{x y}}{\partial x} n_{y}-\frac{\partial v_{x y}}{\partial y} n_{x}\right) \varphi d s- \\
-\oint_{\gamma}\left(v_{x x} \frac{\partial \varphi}{\partial y} n_{y}+v_{y y} \frac{\partial \varphi}{\partial x} n_{x}-v_{x y} \frac{\partial \varphi}{\partial x} n_{y}-v_{x y} \frac{\partial \varphi}{\partial y} n_{x}\right) d s .
\end{gathered}
$$

Подставляя выражения (10) для $v_{i j}$ в равенства $(28),(29)$ и учитывая (21), получим

$$
\begin{gathered}
W_{D} v=-\left(\frac{\partial^{2} \sigma_{x x}}{\partial x^{2}}+\frac{\partial^{2} \sigma_{y y}}{\partial y^{2}}+2 \frac{\partial^{2} \sigma_{x y}}{\partial x \partial y}\right) \\
\left\langle W_{\gamma} v, \varphi=\oint_{\nu}\left[\left(\frac{\partial \sigma_{x x}}{\partial x}+\frac{\partial \sigma_{x y}}{\partial y}\right) n_{x}+\left(\frac{\partial \sigma_{x y}}{\partial x}+\frac{\partial \sigma_{x y}}{\partial y}\right) n_{y}\right] \varphi d s-\right. \\
-\oint_{\nu}\left[\left(\sigma_{x x} n_{x}+\sigma_{x y} n_{y}\right) \frac{\partial \varphi}{\partial x}+\left(\sigma_{x y} n_{x}+\sigma_{y y} n_{y}\right) \frac{\partial \varphi}{\partial y}\right] d s
\end{gathered}
$$

Если уравнение $\left(3_{1}\right)$ продифференцировать по $x$, $\left(3_{2}\right)$ по $y$ и сложить полученные равенства, то будем иметь

$$
\frac{\partial^{2} \sigma_{x x}}{\partial x^{2}}+\frac{\partial^{2} \sigma_{y y}}{\partial y^{2}}+2 \frac{\partial^{2} \sigma_{x y}}{\partial x \partial y}=-\frac{\partial}{\partial z}\left(\frac{\partial \sigma_{x z}}{\partial x}+\frac{\partial \sigma_{y z}}{\partial y}\right)
$$

что в силу $\left(3_{3}\right)$ можно переписать так

$$
\frac{\partial^{2} \sigma_{x x}}{\partial x^{2}}+\frac{\partial^{2} \sigma_{y y}}{\partial y^{2}}+2 \frac{\partial^{2} \sigma_{x y}}{\partial x \partial y}=\frac{\partial^{2} \sigma_{z z}}{\partial z^{2}} .
$$


Сравнивая последнее равенство с (21) и (30), мы видим, что

$$
W_{D} v=0 \text {. }
$$

Согласно $\left(4_{2}\right),\left(4_{3}\right)$, подынтегральное выражение во втором из интегралов, стоящих в правой части равенства (31), тождественно равно нулю, а подынтегральное выражение из первого интеграла согласно $\left(3_{1}\right),\left(3_{2}\right)$ можно преобразовать к виду

$$
\left\langle W_{\nu} v, \varphi\right\rangle=-\oint_{\gamma} \frac{\partial}{\partial z}\left(\sigma_{x z} n_{x}+\sigma_{y z} n_{y}\right) \varphi d s .
$$

Сравнивая это с $\left(4_{3}\right)$, убеждаемся, что $W_{\gamma} v=0$. Вместе с $(27),(32)$ это дает $W v=0$, что и завершает доказательство.

В заключение еще раз отметим, что краевые условия (4) и наши предположения об области $G$ (цилиндр со строго выпуклой кривой $\gamma$ в основании) существенны лишь для определения граничных значений $\left.\sigma_{z z}\right|_{H}$, а соотношения (15)-(17) справедливы для произвольной $G$ безотносительно к краевым условиям.

\section{ПРИЛОЖЕНИЕ}

\section{Обращение лучевого преобразования тензорных полей степени 1 и 2 на плоскости}

Все, что нам нужно, содержится в следствии теоремы 2 работы $\left[{ }^{6}\right]$ или в теореме 3 работы $\left[{ }^{7}\right]$. Тем не менее автор счел нужным поместить настоящее приложение по следующим причинам:

1) для тензорных полей степени 1 и 2 на плоскости (в обозначениях работ $\left[{ }^{6}\right],\left[{ }^{7}\right]$ случай $n=2 ; m=1$ или $m=2$ ) общие формулы, приведенные в этих работах, значительно упрощаются и 2) применение этих результатов несколько затруднено тем обстоятельством, что они получены для тензорных полей, определенных на всей плоскости, а в настоящей работе нас интересует случай поля, заданного в ограниченной области.

Рассмотрим сначала случай векторного поля на плоскости $(n=2$, $m=1)$. Пусть $D-$ плоская область, ограниченная замкнутой $C^{1}$-гладкой кривой $\gamma, u=\left(u_{x}, u_{y}\right)$ - векторное поле, определенное и непрерывное в замкнутой области $D U \gamma$ и имеющее непрерывные частные производные первого порядка в $D$. Будем считать поле $u$ определенным на всей плоскости, положив $u=0$ вне $D \cup \gamma$. Определенное таким образом поле $u$ имеет разрыв на кривой $\gamma$, поэтому входящие в оператор Сен-Венана производные надо понимать в обобщенном смысле. Соответственно этому условимся через $\partial_{x}, \partial_{y}$ обозначать производные в обобщенном смысле, а через $\partial / \partial x, \partial / \partial y$ классические производные. Лучевое преобразование векторного поля на плоскости определяется формулой

$$
I u(x, y, \alpha)=
$$

$=\int_{-\infty}^{\infty}\left[u_{x}(x+t \cos \alpha, y+t \sin \alpha) \cos \alpha+u_{y}(x+t \cos \alpha, y+t \sin \alpha) \sin \alpha\right] d t$.

Оператор Сен-Венана в рассматриваемом случае $(n=2, m=1)$ имеет одну ненулевую компоненту, поэтому мы можем считать, что $W u$ является функцией

$$
W u=\partial_{x} u_{y}-\partial_{y} u_{x}
$$

Ясно, что

$$
W u=W_{D} u+W_{\gamma} u
$$


где

$$
W_{D} u=\left\{\begin{array}{lll}
\frac{\partial u_{y}}{\partial x}-\frac{\partial u_{x}}{\partial y}, & \text { если } & (x, y) \in D, \\
0, & \text { если } & (x, y) \notin D \cup \gamma,
\end{array}\right.
$$

a $W_{\gamma} u$ - обобщенная функция с носителем на $\gamma$, которую мы сейчас вычислим.

Если $f-$ обобщенная функция, а $\varphi-$ пробная (т. е. гладкая финитная) функция, то через $\langle f, \varphi\rangle$ обозначаем значение функционала $f$ на $\varphi$. Согласно определению производных обобщенной функции

$$
\begin{gathered}
\langle W u, \varphi\rangle=\left\langle\partial_{x} u_{y}-\partial_{y} u_{x}, \varphi\right\rangle=\left\langle u_{x}, \frac{\partial \varphi}{\partial y}\right\rangle-\left\langle u_{y}, \frac{\partial \varphi}{\partial x}\right\rangle= \\
=\iint_{D}\left(u_{x} \frac{\partial \varphi}{\partial y}-u_{y} \frac{\partial \varphi}{\partial x}\right) d x d y .
\end{gathered}
$$

Преобразуя последний интеграл по формуле Грина, получим

$$
\langle W u, \varphi\rangle=\iint_{D}\left(\frac{\partial u_{y}}{\partial x}-\frac{\partial u_{x}}{\partial y}\right) \varphi d x d y+\oint_{\gamma}\left(u_{x} n_{y}-u_{y} n_{x}\right) d s,
$$

где $n=\left(n_{x}, n_{y}\right)$ - единичный вектор внешней нормали к $\gamma$. Учитывая (35), это можно переписать в виде

$$
\langle W u, \varphi\rangle=\left\langle W_{D} u, \varphi\right\rangle+\oint_{\gamma}\left(u_{x} n_{y}-u_{y} n_{x}\right) \varphi d s .
$$

Напомним, что $\delta$-функцией, сосредоточенной на кривой $\gamma$, называется обобщенная функция $\delta_{\gamma}$, определяемая равенством

$$
\left\langle\delta_{\gamma}, \varphi\right\rangle=\oint_{\nu} \varphi d s
$$

Равенство (36) означает, что

$$
W u=W_{D} u+\left(u_{x} n_{y}-u_{y} n_{x}\right) \delta_{\gamma} .
$$

Следовательно,

$$
W_{\gamma} u=\left(u_{x} n_{y}-u_{y} n_{x}\right) \delta_{\gamma}
$$

а равенство (34) можно записать в виде

$$
W u=\frac{\partial u_{y}}{\partial x}-\frac{\partial u_{x}}{\partial y}+\left(u_{x} n_{y}-u_{y} n_{x}\right) \delta_{\gamma}
$$

Укажем алгоритм определения $W_{D} u$ по функции $I u(x, y, \alpha)$. Этот алгоритм вытекает из формулы, приведенной в следствии теоремы 2 работы [ $\left.{ }^{6}\right]$ или в теореме 3 работы [ $\left.{ }^{7}\right]$, и состоит в следующем. Сначала определяем векторное поле $\mu=\left(\mu_{x}, \mu_{y}\right)$ на плоскости по формулам

$$
\begin{aligned}
& \mu_{x}(x, y)=\frac{1}{4 \pi} \int_{0}^{2 \pi} I u(x, y, \alpha) \cos \alpha d \alpha \\
& \mu_{y}(x, y)=\frac{1}{4 \pi} \int_{0}^{2 \pi} I u(x, y, \alpha) \sin \alpha d \alpha .
\end{aligned}
$$

Затем находим поле $v=\left(v_{x}, v_{y}\right)$, полагая

$$
v_{x}=(-\Delta)^{1 / 2} \mu_{x} ; \quad v_{y}=(-\Delta)^{1 / 2} \mu_{y},
$$


где $(-\Delta)^{1 / 2}$ - оператор, определяемый с помощью преобразования Фурье $F$ равенствами

$$
F\left[(-\Delta)^{1 / 2} f(x, y)\right]=\varrho \tilde{f}(\xi, \eta) ; \quad \tilde{f}(\xi, \eta)=F[f(x, y)] ; \quad \varrho=\sqrt{\xi^{2}+\eta^{2}} .
$$

Наконец, находим

$$
W_{D} u=W_{D} v=\frac{\partial v_{y}}{\partial x}-\frac{\partial v_{x}}{\partial y}
$$

Второе слагаемое из правой части (34) можно определить по функции $I u$, исходя из той же формулы, но проще это сделать следующим образом. Предположим, что область $D$ строго выпуклая, и пусть $\gamma(s)=$ $=\left(\gamma_{x}(s), \gamma_{y}(s)\right)$ - параметризация кривой $\gamma$ длиной дуги $s$, причем при возрастании $s$ кривая обходится против часовой стрелки. Обозначим через $\alpha(s, \Delta s)$ угол, образуемый с осью $O x$ вектором $\xi(s, \Delta s)=(\gamma(s+\Delta s)-\gamma(s)) /|\gamma(s+\Delta s)-\gamma(s)|$. Тогда

$$
\begin{gathered}
I u\left(\gamma_{x}(s), \gamma_{y}(s), \alpha(s, \Delta s)\right)=\int_{0}^{|\xi|(s, \Delta s)}\left[u_{x}(\gamma(s)+t \xi(s, \Delta s)) \cos \alpha(s, \Delta s)+\right. \\
\left.+u_{y}(\gamma(s)+t \xi(s, \Delta s)) \sin \alpha(s, \Delta s)\right] d t .
\end{gathered}
$$

Отсюда следует, что

$$
\left(u_{x} \dot{\gamma}_{x}+u_{y} \dot{\gamma}_{y}\right)(s)=\lim _{\Delta s \rightarrow 0} \frac{1}{\Delta s} I u\left(\gamma_{x}(s), \gamma_{y}(s), \alpha(s, \Delta s)\right),
$$

где точкой обозначено дифференцирование по $s$. Поскольку $\dot{\gamma}_{x}=$ $=-n_{y}, \dot{\gamma}_{y}=n_{x}$, то предыдущее соотношение можно переписать в виде

$$
\left(u_{x} n_{y}-u_{y} \bar{n}_{x}\right)(s)=-\lim _{\Delta s \rightarrow 0} \frac{1}{\Delta s} I u\left(\gamma_{x}(s), \gamma_{y}(s), \alpha(s, \Delta s)\right) .
$$

Равенства (38), (43) дают выражение $W_{\gamma} u$ через $I u$.

Рассмотрим теперь случай тензорного поля степени 2 на плоскости $(n=m=2)$. Пусть область $D$ такая же, как выше, $v=\left(v_{x x}, v_{x y}, v_{y y}\right)-$ симметричное тензорное поле, определенное и непрерывное вместе с первыми производными в замкнутой области $D U \gamma$ и имеющее непрерывные производные второго порядка в $D$. Полагаем $v=0$ вне $D \cup \gamma$. Лучевое преобразование в данном случае определяется формулой

$$
\begin{gathered}
I v(x, y, \alpha)=\int_{-\infty}^{\infty}\left[v_{x x}(\bar{x}) \cos ^{2} \alpha+2 v_{x y}(\bar{x}) \cos \alpha \sin \alpha+\right. \\
\left.+v_{y y}(\bar{x}) \sin ^{2} \alpha\right]_{\bar{x}=(x+t \cos \alpha, y+t \sin \alpha)} d t .
\end{gathered}
$$

Оператор Сен-Венана в случае $n=m=2$ имеет одну ненулевую компоненту

$$
W v=2 \partial_{x y} v_{x y}-\partial_{y y} v_{x x}-\partial_{x x} v_{y y} .
$$

C помощью рассуждений, аналогичных использованным при выводе (39), легко показать, что

$$
W v=W_{D} v+W_{v} v,
$$

где

$$
W_{D} v(x, y)=\left\{\begin{array}{l}
2 \frac{\partial^{2} v_{x y}}{\partial x \partial y}-\frac{\partial^{2} v_{x x}}{\partial y^{2}}-\frac{\partial^{2} v_{y y}}{\partial x^{2}}, \text { если } \quad(x, y) \in D, \\
0, \text { если }(x, y) \notin D \cup \gamma,
\end{array}\right.
$$


a $W_{\gamma} v$ - обобщенная функция с носителем на $\gamma$, определяемая равенством

$$
\begin{gathered}
\left\langle W_{\nu}, \varphi\right\rangle=\oint_{\nu}\left[\left(\frac{\partial v_{y y}}{\partial x}-\frac{\partial v_{x y}}{\partial y}\right) n_{x}+\left(\frac{\partial v_{x x}}{\partial y}-\frac{\partial v_{x y}}{\partial x}\right) n_{y}\right] \varphi d s- \\
-\int_{\nu}\left[\left(v_{y y} n_{x}-v_{x y} n_{y}\right) \frac{\partial \varphi}{\partial x}+\left(v_{x x} n_{y}-v_{x y} n_{x}\right) \frac{\partial \varphi}{\partial y}\right] d s .
\end{gathered}
$$

Алгоритм определения $W_{D} v$ по $I v$ состоит в следующем. Сначала находим тензорное поле $\mu=\left(\mu_{x x}, \mu_{x y}, \mu_{y y}\right)$ на плоскости по формулам

$$
\begin{aligned}
& \mu_{x x}(x, y)=\frac{1}{4 \pi} \int_{0}^{2 \pi} I v(x, y, \alpha) \cos ^{2} \alpha d \alpha \\
& \mu_{x y}(x, y)=\frac{1}{4 \pi} \int_{0}^{2 \pi} I v(x, y, \alpha) \cos \alpha \sin \alpha d \alpha \\
& \mu_{y y}(x, y)=\frac{1}{4 \pi} \int_{0}^{2 \pi} I v(x, y, \alpha) \sin ^{2} \alpha d \alpha .
\end{aligned}
$$

Затем определяем поле $v=\left(v_{x x}, v_{x y}, v_{y y}\right)$ равенствами

$$
\begin{gathered}
v_{x x}=(-\Delta)^{1 / 2}\left(\mu_{x x}-\frac{1}{2} \mu_{y y}\right), \quad v_{x y}=\frac{3}{2}(-\Delta)^{1 / 2} \mu_{x y}, \\
v_{y y}=(-\Delta)^{1 / 2}\left(\mu_{y y}-\frac{1}{2} \mu_{x x}\right) .
\end{gathered}
$$

Наконец, полагаем

$$
W_{D} v=W_{D} v=2 \frac{\partial^{2} v_{x y}}{\partial x \partial y}-\frac{\partial^{2} v_{x x}}{\partial y^{2}}-\frac{\partial^{2} v_{y y}}{\partial x^{2}}
$$

Можно выразить через Iv и второе слагаемое из правой части (44), однако мы здесь этого делать не будем.

В настоящее время автор не имеет опыта вычислений по формулам $(40)-(42)$ и $(47)-(49)$ и потому не может сказать, насколько они приемлемы с точки зрения практического применения. Возможно, что для этой цели их надо предварительно каким-либо образом преобразовать. В связи с этим отметим, что эти формулы по своей структуре во многом аналогичны формуле обращения преобразования Радона (ср. с теоремой 3.1 из $\left[{ }^{9}\right]$ в случае $n=2$ ), поэтому здесь можно воспользоваться опытом, накопленным в томографии $\left[{ }^{10}\right]$.

Автор благодарит X. Абена за обсуждение предмета работы.

\section{Л И Т Е РА Т У Р А}

1. Абен X. К. Интегральная фотоупругость. Таллинн, Валгус, 1975.

2. Leray, T., Scheibling, G. // J. Chim. Phys. (France), 1961, 68, № 9, 797-802.

3. Абен Х. К., Индурм С. И., Иозепсон Ю. И., Келл К.-Ю. Э. // Оптическая томография. Всесоюзн. сем., Таллинн, 1988. Тез. докл. Таллинн, 1988, 7.

4. Шарафутдинов B. A. // Сиб. мат. журн., 1983, 24, № 6, 176-187. 
5. Шарафутдинов В. А. // Докл. АН СССР, 1986, 286, № 2, 305-307.

6. Шарафутдинов В. А. // Докл. АН СССР, (в печати).

7. Шарафутдинов B. A. // Всесоюзн. сем. по оптической томографии, Таллинн, апрель 1988. Тез. докл., Таллинн, $1988,160$.

8. Cheng, Y. F. // Exp. Mech., 1970, 10, № 2, 534-536.

9. Хелгасон С. Преобразование Радона. М., Мир, 1983.

10. Хермен $\Gamma$. Восстановление изображений по проекциям. Основы реконструктивной томографин. М., Мир, 1983.

Институт математики Сибирского отделения Академии наук СССР

Поступила в редакцию

20/II 1989

\section{SARAFUTDINOV}

\section{INTEGRAALNE FOTOELASTSUS NORGA OPTILISE ANISOTROOPIA KORRAL}

On vaadeldud pingete määramist silindrilises $z$-teljega kehas nende mõõtmistulemuste pōhjal, mis on saadud pinnaga $z=0$ paralleelsetel sirgetel. On eeldatud, et keha optiline anisotroopia on nõrk, ja näidatud, et niisugusel juhul saab määrata vaid pingetensori komponendi $\sigma_{z z}$.

\section{SHARAFUTDINOV}

\section{ON INTEGRATED PHOTOELASTICITY IN CASE OF WEAK BIREFRINGENCE}

Determination of stress in a cylindrical body with axis $z$ on the basis of experimental data measured along all the lines parallel to the plane $z=0$, is considered on the assumption that birefringence is weak. It is shown that in this case it is possible to determine only the component $\sigma_{z z}$ of the stress tensor. 